\title{
Case Series Analysis of New Zealand Reports of Rapid Intense Potentiation of Warfarin by Roxithromycin
}

\author{
Ruth L. Savage ${ }^{1,2} \cdot$ Michael V. Tatley ${ }^{1}$
}

Published online: 12 January 2018

(c) The Author(s) 2018. This article is an open access publication

\begin{abstract}
Introduction We undertook an analysis of all the reports to the New Zealand Centre for Adverse Reactions Monitoring of a roxithromycin/warfarin interaction after two recent reports described intense rapid warfarin potentiation. The interaction was first published in 1995. Cytochrome P450 3A4 inhibition has been the proposed mechanism but has limited biologic plausibility. There are suggestions that the clinical significance of the interaction may be increased by severe illness, polypharmacy, renal dysfunction, older age and increased warfarin sensitivity.

Methods To investigate the potentiating effect of warfarin on roxithromycin in this New Zealand case series, the reports were reviewed to identify patients at risk, compare the reporting pattern with published Australian data and evaluate the appropriateness of current prescribing advice. Results Thirty patient reports were identified. The age range was 23-88 years, mean 66.8, median 73.0 (standard deviation 17.7) and the international normalised ratios after roxithromycin commencement ranged from 3.6 to 16.7 (mean 7.6, median 7.6, standard deviation 3.6). For eight patients with measurements on day 3 , international normalised ratios were 4.3-16.7 (mean 10.4, median 8.8, standard deviation 4.4). Four patients had serious haemorrhage. Indications for roxithromycin were a range of respiratory tract infections. Anticoagulation was stable for most patients prior to acute infection.
\end{abstract}

Ruth L. Savage

ruth.savage@otago.ac.nz

1 New Zealand Pharmacovigilance Centre, Dunedin School of Medicine, University of Otago, PO Box 913, Dunedin 9054, New Zealand

2 Department of General Practice, University of Otago, Christchurch, New Zealand
Serious infection occurred in 54.5\% (12 of 22 patients with information). Polypharmacy (five or more medicines daily) was used by $36.7 \%$ of patients long term, increasing acutely to $83.3 \%$, including additional potentially interacting medicines. Warfarin daily dose (1.5-13.0 mg, mean 4.4, median 4.0, standard deviation 2.2) was moderate to low. Pre-roxithromycin international normalised ratio values ranged from 1.4 to 3.7 , mean and median 2.5 , standard deviation 0.5 . A high proportion of interactions were observed between warfarin and roxithromycin compared with other macrolides and compared with cytochrome P450 3A4-related macrolide interactions. The pattern was similar to published Australian data.

Conclusion In this case series, the high prevalence of acute polypharmacy, including potentially interacting medicines, and serious infection suggests that they may have contributed to warfarin potentiation and increased the clinical significance of a roxithromycin/warfarin interaction.

\section{Key Points}

In this study of a clinically important interaction observed between roxithromycin and warfarin in patients predominantly aged over 65 years, there was a high prevalence of acute polypharmacy and serious infection

Despite previous assumptions, inhibition of the cytochrome P450 enzyme is unlikely to explain the rapid and intense potentiation of warfarin observed

The findings support international normalised ratio measurement within 3 days when roxithromycin is added to warfarin and limiting roxithromycin in patients taking warfarin to infections for which macrolides are first choice. 


\section{Introduction}

Roxithromycin is a macrolide antibiotic with an antibacterial spectrum of activity similar to that of erythromycin but with a longer half-life, which allows for twice-daily dosing [1]. When roxithromycin became available, erythromycin was already known to interact with warfarin but a small study of healthy volunteers had not demonstrated an interaction between roxithromycin and warfarin [2]. Since then, roxithromycin has not been well represented in studies of warfarin interactions with antibiotics. However, evidence for an interaction was first published in 1995 as a case series from New Zealand and Australia [3]. Reporters were aware of the interaction between erythromycin and warfarin and shared their concern that roxithromycin appeared to be interacting similarly.

Erythromycin is known to be a potent cytochrome P450 (CYP)3A4 inhibitor and roxithromycin a weak inhibitor [4]. It was assumed that roxithromycin is therefore less likely to interact with warfarin [3]. However, only (R)warfarin, the less active enantiomer, is a substrate for CYP3A4, thus inhibition of its metabolism by this mechanism appears an unlikely explanation for rapid intense warfarin potentiation with roxithromycin observed in ongoing reports to the New Zealand Centre for Adverse Reactions Monitoring (CARM) since the 1995 publication. For example, two recent reports describe international normalised ratios (INRs) of 16.1 and 8.4 observed as early as day 3 and 4 after roxithromycin was added to warfarin treatment [5, 6]. In support of these observations, in 2015, an Australian study identified 72 anticoagulated elderly patients who required vitamin $\mathrm{K}$ administration because of INR values $>10$. Five of these had taken roxithromycin with warfarin [7].

It has been suggested that the interaction may be more clinically significant with polypharmacy, severe illness, renal dysfunction, and in those who are elderly or otherwise at risk of increased warfarin sensitivity $[3,7,8]$. Patients taking low doses of warfarin may be most at risk as clearance is already low [9]. Infection, antibiotic use in general and high target INR values have been shown to potentiate warfarin activity in some studies $[10,11]$.

The origins of recent reports for roxithromycin in VigiBase, the World Health Organization Global Database for Individual Case Safety Reports [12], indicate that it is used in Australia, New Zealand, Asia, including China and India, and parts of Europe. It is not marketed in the UK or USA, which may, in part, explain why it is not well represented in studies of antibiotic interactions with warfarin. Development of prescribing advice is challenging because of the paucity of information about the mechanisms and risk factors for the interaction. There is also limited information regarding whether roxithromycin differs from other macrolides with regard to intensity and frequency of the interaction or how macrolides compare with other antibiotics.

We present an analysis of a case series of 30 reports of an interaction between warfarin and roxithromycin, a macrolide antibiotic. They represent all the reports submitted to CARM for this interaction from when the first report was received in 1992 until December 2015. In the absence of formal studies, the objective of this study was to analyse all reports of an interaction between warfarin and roxithromycin submitted to CARM to identify patients at risk, compare the reporting pattern with published Australian data [13] and evaluate the appropriateness of current prescribing advice.

\section{Methods}

All reports of an interaction between warfarin and roxithromycin submitted to CARM until December 2015 were reviewed for patient demographics, therapeutic indications, co-morbidities, concomitant medicines and warfarin dose. Warfarin duration and INR values prior to roxithromycin were reviewed to assess anticoagulant stability. Because infection decreases warfarin requirements, we attempted to identify patients with serious infection. Patients with pneumonia and those hospitalised for infection were considered to have serious infection. Concomitant medicines that were listed in Stockley's Interaction Alerts as requiring increased INR monitoring or consideration of increased monitoring if they were prescribed with warfarin were noted [9]. They were considered potentially interacting in the case series unless their use was described as "long term" or exceeded 2 months.

The minimum number of medicines used to define 'polypharmacy' generally ranges from 5 to 10 [14]. Polypharmacy was defined as five or more medicines daily in this study. Patients whose warfarin use was 2 months or more, or was described as "long term", were considered long-term users. The characteristics of patients with an INR value $>8$, a cut-off point for vitamin $\mathrm{K}$ treatment [15], were also considered separately. Statistical analysis between groups was not conducted because of small numbers.

To consider the potential mechanism, the frequency of reporting of a roxithromycin interaction with warfarin was compared with CYP3A4-related roxithromycin interactions and corresponding interactions for all macrolides in the CARM database. A comparison was made with published Australian pharmacovigilance data [13]. 


\section{Results}

We found 30 reports of an interaction between warfarin and roxithromycin in CARM with the first report received in 1992. The INR range after roxithromycin was added to warfarin was 3.6-16.7 [mean 7.6, median 7.6, standard deviation (SD) 3.6] (Table 1). Figure 1 shows the INR values by day of roxithromycin treatment. International normalised ratio values for eight patients measured on day 3 of roxithromycin treatment were 4.3-16.7, mean and median 10.4 and 8.8, SD 4.4. Twelve patients experienced haemorrhage, four were serious events. The male-to-female ratio was 9:21 but this increased with age to $1: 1$ in patients aged over 80 years. The age range was 23-88 years, mean 66.8, median 73.0, SD 17.7. Although a predominantly elderly group, seven patients $(23.3 \%)$ were aged $<65$ years. The other parameters studied are shown in Table 1 and discussed below.

\subsection{Pre-Roxithromycin Anticoagulant Stability}

Table 1 shows information on duration of warfarin use reported for $24(80 \%)$ patients. Twenty-one patients were long-term warfarin users with no indication of a recent dose increase. Three patients were short-term users or had recently increased the dose. Table 1 also shows pre-roxithromycin INRs, which were reported for $20(67 \%)$ patients and ranged from 1.4 to 3.7 (mean and median 2.5, SD 0.5). Fourteen reports indicated that the INR had been measured within a month of starting roxithromycin. A range of INR values over specific time periods was reported for six patients. Two to four readings for each patient were taken over 2-6 weeks. Out of a total of 20 readings, 17 were in the therapeutic range (1.5-3.0). The remaining 14 patients had one or two INR readings prior to roxithromycin and only two patients were outside the therapeutic range. Four reports with one or two INRs and one with no INR values indicated that the patients' anticoagulation was usually stable. One of these indicated an average of 2.6 over 18 months and no change in warfarin dose over the most recent 3 months.

\subsection{Potentially Interacting Medicines (in Addition to Roxithromycin)}

Potentially interacting medicines were taken by nine patients. They took high-dose prednisone (5), amiodarone (2), tramadol (2), one with prednisone, or allopurinol (1) short term or for an unknown duration. Five of these nine patients were also taking one or more antibiotics, cephalosporins, amoxicillin/clavulanate, doxycycline or co-

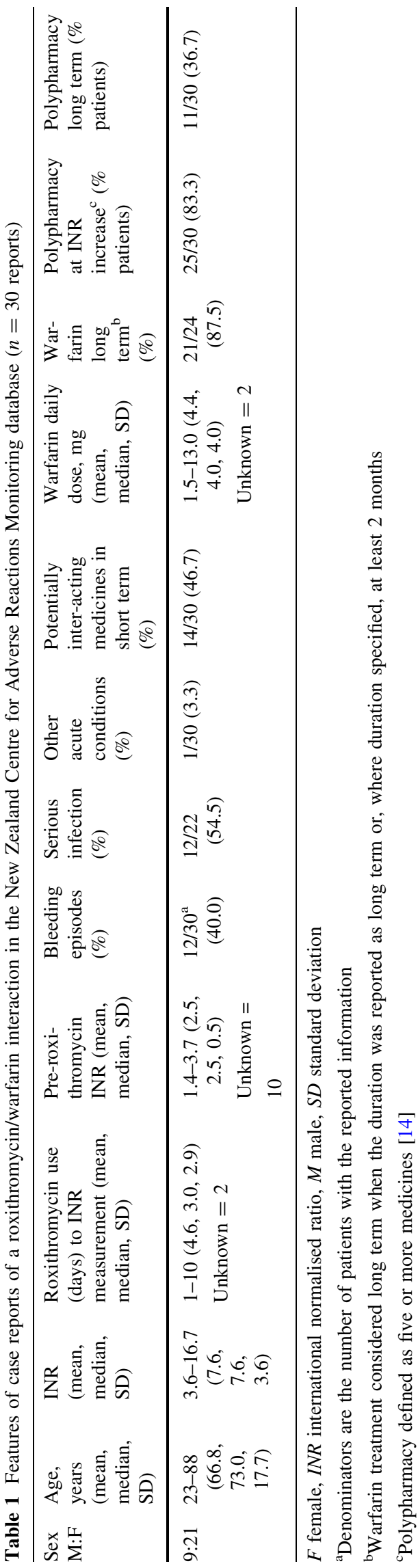


Fig. 1 International normalised ratio (INR) value by day after roxithromycin added to warfarin

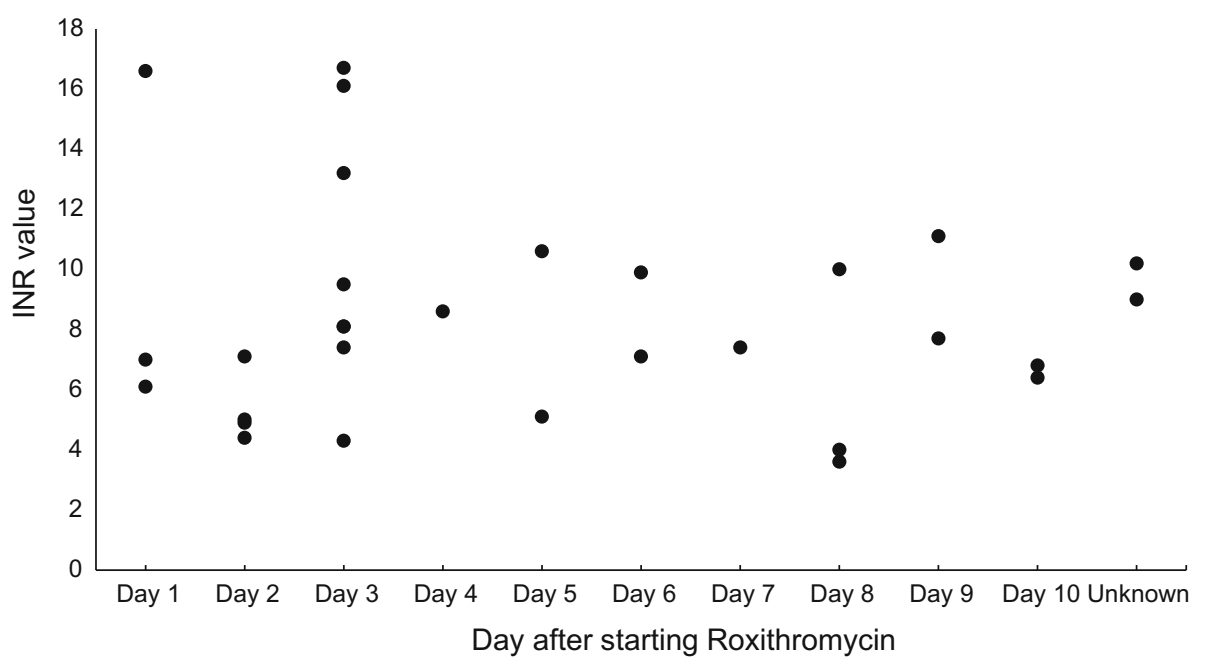

trimoxazole. A further five patients took short-term cefuroxime, amoxicillin or amoxicillin clavulanate only. The effect of these is uncertain as relevant interactions seem very rare [9].

\subsection{Polypharmacy}

A high percentage of patients $(83.3 \%)$ were taking five or more medicines when the INR increased after roxithromycin was added to warfarin. However, prior longterm polypharmacy was considerably less at $36.7 \%$. Patients were prescribed between one and four additional medicines around the time roxithromycin was commenced. The majority of the medicines were oral prescription medicines. Five medicines were inhaled bronchodilators or corticosteroids (3), a mineral supplement and a vitamin supplement but the patients taking these were also taking at least five oral prescription medicines.

\subsection{Serious Infection}

The indications for roxithromycin were upper and lower respiratory infections. Because of limited information, seriousness of infection could only be assessed for 23 of the patients but $14(60.9 \%)$ had serious infection as indicated by a diagnosis of pneumonia and/or hospital admission. Consequently, at least $47 \%$ of the 30 patients in the case series had serious infection.

\subsection{Acute Co-morbidities}

Only one patient was reported to have another acute illness, acute cardiac failure, in addition to the indication for roxithromycin. No patients were reported to be in renal or hepatic failure.

\subsection{Warfarin Sensitivity}

The daily warfarin dose was $1.5-13.0 \mathrm{mg}$ (mean 4.4, median 4.0, SD 2.2). Joffe et al. [16] classified daily warfarin doses as low $\leq 2 \mathrm{mg}$, moderate, $4-6 \mathrm{mg}$, and high $\geq 10 \mathrm{mg}$. The mean daily dose in our case series therefore appears moderate to low. One patient was taking $\leq 2 \mathrm{mg}$ and one $\geq 10 \mathrm{mg}$. The lower doses would have been influenced by age because the mean daily dose for patients aged $\geq 75$ years was $3.2 \mathrm{mg}$ compared with $5.2 \mathrm{mg}$ for patients aged $<75$ years. There was no suggestion that the patients were a group particularly sensitive to warfarin before the onset of a respiratory infection and treatment with roxithromycin.

\subsection{Clinical Profiles}

Approximately two-thirds of patients (19/30) had serious infection, acute co-morbidities or took interacting medicines or combinations of these. Of the remaining 11 patients, five had insufficient information and six had no obvious contributors to increased INRs apart from roxithromycin.

\subsection{Subgroup Observations}

Seven patients had INR values $>8.0$ measured within 3 days of roxithromycin treatment. The number of patients was small for comparison but there was evidence of a trend towards a greater prevalence of serious infection in the rapid-onset high-INR group, 4/5 (80.0\%), compared with $12 / 22(54.5 \%)$ in the whole cohort, the denominators being the number of patients with information about infection.

\subsection{Individual Case Reports}

It could be argued that some of the reports provide alternative explanations to roxithromycin for the warfarin 
Table 2 Interactions with macrolides in reports to the New Zealand Centre for Adverse Reactions Monitoring (CARM) and the Australian Adverse Drug Reactions Advisory Committee (ADRAC) [13]

\begin{tabular}{llllll}
\hline Drug & $\begin{array}{l}\text { Interacting } \\
\text { drug }\end{array}$ & $\begin{array}{l}\text { No, of interaction reports, } \\
\text { CARM 1992-2015 }\end{array}$ & $\begin{array}{l}\text { No. of interaction reports, } \\
\text { ADRAC 1995-2004 }\end{array}$ & $\begin{array}{l}\text { Total reports for } \\
\text { macrolides, CARM }\end{array}$ & $\begin{array}{l}\text { Total reports for } \\
\text { macrolides, ADRAC }\end{array}$ \\
\hline \multirow{2}{*}{ Warfarin } & Roxithromycin & 30 & 53 & 270 & 737 \\
& Clarithromycin & 0 & 7 & 53 & 193 \\
& Erythromycin & 4 & 6 & 777 & 597 \\
& Azithromycin & 0 & 4 & 43 & 111 \\
\multirow{5}{*}{ Anticonvulsants } & Roxithromycin & 0 & 5 & 270 & 737 \\
& Erythromycin & 4 & 3 & 777 & 597 \\
\multirow{5}{*}{ Statins } & Clarithromycin & 0 & 2 & 53 & 193 \\
& Roxithromycin & 3 & 5 & 270 & 777 \\
& Erythromycin & 14 & 4 & 53 & 597 \\
& Clarithromycin & 0 & 2 & & 193 \\
\hline
\end{tabular}

potentiation. Two patients had INR values of 16.6 and 7.0 on the day after roxithromycin was commenced. Both had received tramadol the day prior to roxithromycin, one with high-dose prednisone and cefuroxime and the other with gentamicin, suggesting serious infection. In these case reports, the relative contribution of each medicine and clinical condition is unclear. However, other reports do suggest that roxithromycin was an important contributor. A 63-year old patient had an INR of 16.1 on day 3 of roxithromycin treatment with no other apparent contributing factors. A patient aged 25 years with rheumatic heart disease developed pneumonia with an INR increase from 2.2 to 3.7. She was treated with roxithromycin and after 3 days her INR was 13.2. One reporter indicated that their patient had recurrent infective exacerbations of chronic obstructive pulmonary disease but these had never resulted in an increased INR until the most recent episode when the patient was treated with roxithromycin.

\section{Roxithromycin and Other Macrolide Interactions in New Zealand and Australia}

Table 2 shows the profile of the most frequently reported interactions with macrolides from the New Zealand CARM and published by the Australian Adverse Drug Reactions Advisory Committee [13], revealing a marked similarity between the two countries. There is prominent reporting of a roxithromycin/warfarin interaction compared with other macrolides and warfarin and compared with other roxithromycin interactions. Of the reports for roxithromycin in the Australian database, more than $10 \%$ were for interactions, predominantly with warfarin. With regard to the interactions with anticonvulsants and statins which are CYP3A4 dependent, the Australian Adverse Drug
Reactions Advisory Committee concluded that the mechanism for the interaction between roxithromycin and warfarin probably did not involve CYP3A4.

\section{Discussion}

The case series described here identified a pattern of patients with predominantly stable warfarin anticoagulation who developed an acute infection, often serious, and/ or were treated with several medicines, some potentially interacting, including roxithromycin, before marked warfarin potentiation occurred. The warfarin dose suggests that some patients had increased warfarin sensitivity and this may have been influenced by older age. However, younger patients were also affected with seven (23.3\%) aged $<65$ years. Polypharmacy (five or more daily medicines) increased around the time of roxithromycin prescription to over $80 \%$ but less than $40 \%$ of patients were exposed to long-term polypharmacy. There was no evidence that renal or hepatic dysfunction, multiple co-morbidities, high target INR values or low warfarin doses were important contributors. At least six of the 30 patients (20\%) had no apparent reason for the increased INR except roxithromycin and non-serious infection. Limitations are lack of information on adherence, which may be affected by acute infection, and incomplete data on time within the therapeutic range prior to roxithromycin treatment.

The reported patterns of roxithromycin interactions in the New Zealand and Australian pharmacovigilance databases (Table 2) do not add to our knowledge about the mechanism, although they suggest that CYP3A4 inhibition is unlikely to be the mechanism. This is in keeping with in vitro studies demonstrating only weak inhibition of $(R)$ warfarin-7-hydroxylation by roxithromycin using 
recombinant CYP3A4. The same studies did not show any inhibition of $(R)$ - and $(S)$-warfarin 7-hydroxylation by roxithromycin using recombinant CYP2C9 [4], which has been proposed as an alternative pharmacokinetic explanation [13].

Prescribing advice is difficult to formulate for warfarin and roxithromycin and, indeed, for other macrolides and antibiotics where there is limited information about the frequency and clinical significance of the interaction and the conditions for which they are indicated are likely to affect anticoagulation. The 2012 American College of Chest Physicians' publication of evidence-based clinical practice guidelines for the management of anticoagulant therapy commented that data addressing interactions between antibiotics and warfarin from multiple large database studies presented a somewhat confusing picture. The authors considered that there were sufficient studies to suggest an increased risk with co-trimoxazole, which inhibits CYP2C9, and quinolones but other groups implicated had been insufficiently studied [17]. Since then, two ambulatory care studies, one for patients with acute upper respiratory tract infection, found that 3.8 and $16 \%$ of patients developed INR values of $\geq 5.0$ and $>4.0$, respectively when the macrolide, azithromycin, was added to warfarin treatment $[18,19]$. This places azithromycin at an intermediate-risk level along with other antibiotics that appear to potentiate warfarin without interacting at a pharmacokinetic level. The patients in these studies are representative of the patients who were not seriously ill in our case series for roxithromycin. For another macrolide, they provide a baseline frequency for patients likely to experience an interaction, which may well increase when more unwell patients are treated.

Cautions about combined roxithromycin and warfarin use are only partly consistent. The interaction appears in product information we obtained from four countries [20-24], with advice to monitor, though not at any specific time. In the Swedish Summary of Product Characteristics [21], the combination is not recommended and one sponsor in New Zealand includes it in the Special Warnings and Precautions section. [22] In the remaining Summaries of Product Characteristics, it only appears under Interactions. The Interaction sections in all the countries commence with a comment about the lower affinity of roxithromycin for CYP than erythromycin and this may lead prescribers to consider roxithromycin a safer option for patients taking warfarin.

Baillargeon et al. based on the assumption that antibiotic interactions with warfarin are due to CYP inhibition or alteration in vitamin-K producing intestinal flora leading to an increased INR and hence increased bleeding within a 1to 2-week period, recommended monitoring the INR 1 week after initiating antibiotic therapy or more frequently for patients at a higher risk of bleeding. [19] However, after considering case reports, the New Zealand medicines regulator, Medsafe, recommended that the INR should be measured on day 3 after macrolides are added to warfarin treatment. [25] This is in keeping with Stockley's Medicine Interaction Alerts [9], which recommends measurement within 3 days for antibiotics with some evidence of an interaction. It is likely, from the evidence in our case series, that this advice has prevented haemorrhage by avoiding prolonged intense warfarin potentiation, especially in patients susceptible to bleeding such as the elderly. The high INR values obtained on days $1-3$ of roxithromycin treatment in our case series also make it clear that the clinical practice of measuring the INR daily in seriously ill patients is crucial.

There appears to have been less discussion about minimising potential harm by considering the indications for roxithromycin. It is approved for a wide range of upper and lower respiratory tract infections and a small number of other infections. The indications in our case series were a range of respiratory infections. In response to the Australian study of elderly patients presenting for vitamin $\mathrm{K}$ administration [7], Thompson commented on the wide use of roxithromycin in Australia beyond national guidelines [26]. In the 2017 New Zealand antibiotic guideline for respiratory infection [27], macrolides are first-choice antibiotics only for atypical pneumonia, pertussis infection and, if penicillin allergic, other pneumonias and group A haemolytic streptococcal pharyngitis. It would be appropriate to limit prescribing of roxithromycin for patients taking warfarin to infections that local guidelines indicate are most appropriately treated with macrolides. This would avoid unnecessary risk in patients who may well respond to antibiotics less likely to interact, in particular those with conditions such as exacerbations of chronic obstructive pulmonary disease where potentially interacting high-dose corticosteroids are also prescribed.

Currently, the advice applies equally to the macrolides. There is reasonable evidence for erythromycin and azithromycin. However, there is less evidence for clarithromycin [6]. More study is needed to evaluate the effect of individual macrolides on the activity of warfarin and the wider group of vitamin $\mathrm{K}$ antagonists. Large observational studies have provided little information about individual macrolides. Case reports in VigiBase, if followed up for detail as in this case series, could be a useful source of information despite the uneven nature of individual case safety reporting.

\section{Conclusion}

The case series is characterised by a high prevalence of polypharmacy and serious infection. Acute anticoagulation destabilisation with severe infection and additional 
interacting medicines may therefore have increased the clinical significance of a roxithromycin/warfarin interaction. Warfarin sensitivity may have contributed influenced by older age. There was no evidence that renal dysfunction, multiple co-morbidities and a high target INR contributed. The findings reinforce the low likelihood that the interaction is CYP3A4 mediated. The challenge of developing prescribing advice for antibiotic use with warfarin is noted. Advice to measure the INR within 3 days after starting roxithromycin treatment is reinforced and should be promoted. It is suggested that the risk of harm could be further minimised by limiting prescribing of roxithromycin for patients taking warfarin to infections for which macrolide antibiotics are first choice.

\section{Compliance with Ethical Standards}

Funding No sources of funding were used to assist in the preparation of this study.

Conflicts of interest Ruth L. Savage and Michael V. Tatley have no conflicts of interest directly relevant to the content of the study.

Ethics Approval The researchers are members of the New Zealand national adverse drug reaction monitoring service who performed this analysis based on observations arising from routine review of voluntary adverse reaction reports submitted to the New Zealand pharmacovigilance database. Ethical approval is not required for national pharmacovigilance database review activities.

Open Access This article is distributed under the terms of the Creative Commons Attribution-NonCommercial 4.0 International License (http://creativecommons.org/licenses/by-nc/4.0/), which permits any noncommercial use, distribution, and reproduction in any medium, provided you give appropriate credit to the original author(s) and the source, provide a link to the Creative Commons license, and indicate if changes were made.

\section{References}

1. Drugdex. Roxithromycin. http://www.micromedexsolutions.com. Accessed 21 Dec 2017.

2. Paulsen O, Nilsson L, Saint-Salvi B, et al. No effect of roxithromycin on pharmacokinetic or pharmacodynamic properties of warfarin and its enantiomers. Pharmacol Toxicol. 1988;63:215-20.

3. Ghose K, Ashton J, Rohan A. Possible interaction of roxithromycin with warfarin: updated review of ADR reports. Clin Drug Investig. 1995;10:302-9.

4. Yamazaki H, Shimada T. Comparative studies of in vitro inhibition of cytochrome P450 3A4-dependent testosterone 6b-hydroxylation by roxithromycin and its metabolites, trolandeomycin and erythromycin. Drug Metab Dispos. 1998;26:1053-7.

5. The Centre for Adverse Reactions Monitoring. Dunedin: University of Otago; 2017. http://nzphvc.otago.ac.nz. Accessed 11 Dec 2017.

6. Medsafe. Suspected Medication Adverse Reactions Search (SMARS). Medsafe; 2017. http://www.medsafe.govt.nz/Projects/ B1/ADRDisclaimer.asp. Accessed 6 Nov 2017.
7. Rotella J-A, Taylor DM. Concomitant roxithromycin use amongst elderly patients taking warfarin resulting in significant over-anticoagulation. J Pharm Pract Res. 2015;45:182-5.

8. Medsafe editorial team. Watching briefs. Roxithromycin-warfarin interaction of increased INR. Prescriber update 2008; 29:2. http:// www.medsafe.govt.nz/profs/PUArticles/watchingbriefsMay08. htm\#Roxithromycin. Accessed 10 Jun 2017.

9. Stockley's interaction alerts. http://nzf.org.nz/interactions/ stockleys/of/10249701000116101. Accessed 30 Sep 2017.

10. Panneerselvam S, Baglin C, Lefort W, Baglin T. Analysis of risk factors for over-anticoagulation in patients receiving long-term warfarin. Br J Haematol. 1998;103:422-4.

11. Glasheen JJ, Fugit RV, Prochazka AV. The risk of overanticoagulation with antibiotic use in outpatients on stable warfarin regimens. J Gen Intern Med. 2005;20:653-6.

12. Lindquist M. VigiBase, the WHO Global ICSR Database System: basic facts. Drug Inf J. 2008;42:409-19.

13. ADRAC. Interactions with macrolides. Aust Adv Drug React Bull. 2006;25:7-8.

14. Rochon PA, Schmader KE, Sullivan DJ. Drug prescribing for older adults. 26 May 2017. http://www.uptodate.com/contents/ drug-prescribing-for-older-adults. Accessed 20 Sep 2017.

15. Keeling D, Baglin T, Tait C, Watson H, Perry D, Baglin C, et al. British committee for standards in haematology. Br J Haematol. 2011;154:311-24.

16. Joffe HV, Xu R, Bradley Johnson F, et al. Warfarin dosing and cytochrome P450 2C9 polymorphisms. Thromb Haemost. 2004;91:1123-8.

17. Holbrook A, Schulman S, Witt DM, et al. Evidence-based management of anticoagulant therapy. Antithrombotic therapy and prevention of thrombosis. 9th ed. American College of Chest Physicians evidence-based clinical practice guidelines. Chest. 2012;141(2 Suppl.):152-84.

18. Clark NP, Delate T, Riggs CS, et al. Warfarin interactions with antibiotics in the ambulatory care setting. JAMA Intern Med. 2014;174:409-16.

19. Baillargeon J, Holmes HM, Lin Y-L, Raji MA, Sharma G, Kuo Y-F. Concurrent use of warfarin and antibiotics and the risk of bleeding in older adults. Am J Med. 2012;125:183-9.

20. Sanofi-Aventis, France. Rulid. 23 May 2017. http://base-donneespublique.medicaments.gouv.fr/affichageDoc.php?specid= 65393957\&typedoc=R. Accessed 15 Sep 2017.

21. Sanofi AB. Surlid. 2 July 2017. http://www.fass.se/LIF/ product ? \&userType $=0 \&$ nplId $=19900405000019 \&$ docType $=6$. Accessed 15 Sep 2017.

22. Teva Pharma New Zealand. Arrow-Roxithromycin. 10 Feb 2017. http://www.medsafe.govt.nz/profs/datasheet/a/ ArrowRoxithromycintab.pdf. Accessed 15 Sep 2017.

23. Sanofi-Aventis New Zealand Ltd. Rulide. 8 Sep 2017. http:// www.medsafe.govt.nz/profs/Datasheet/r/rulideDtab.pdf. Accessed 15 Sep 2017.

24. Sanofi-Aventis Australia Pty Ltd. Rulide. 21 Jan 2015. http:// www.ebs.tga.gov.au/ebs/picmi/picmirepository.nsf/ pdf?OpenAgent $\&$ id=CP-2010-PI-06117-3\&d= 2016050416114622483. Accessed 15 Sep 2017.

25. Medsafe editorial team. Check INR after starting roxithromycin for patients on warfarin. Prescriber update. 2015; 36:3. http:// www.medsafe.govt.nz/profs/PUArticles/ March2015Roxithromycin.htm. Accessed 10 Jun 2017.

26. Thompson A. Concomitant roxithromycin use amongst elderly patients taking warfarin resulting in significant over-anticoagulation (letter to the editor). J Pharm Pract Res. 2015;45:486.

27. BPAC $^{\mathrm{nz}}$. The $2017 \mathrm{bpac}^{\mathrm{nz}}$ antibiotic guide: choices for common infections. www.bpac.org.nz/antibiotics/guide.aspx. Accessed 16 Sep 2017. 